\title{
A HOLLING TYPE II FOOD CHAIN SYSTEM WITH BIOLOGICAL AND CHEMICAL CONTROLS
}

\author{
HUNKI BAEK
}

\begin{abstract}
For a class of Holling type II food chain systems with biological and chemical controls, we give conditions of the local stability of prey-free periodic solutions and of the permanence of the system. Further, we show the system is uniformly bounded.
\end{abstract}

\section{Introduction}

There are number of factors in the environment to be considered in population models. One of important factors is impulsive perturbation such as fire, flood, etc, that are not suitable to be considered continually. These impulsive perturbations bring sudden change to the system. For example, consider the human artificial activities to control the density of the prey and regard the prey as a pest. There are many ways to beat pests such as biological or chemical tactics. Biological control is to reduce the pest population using the actions of other living organisms, often called natural enemies or beneficial species. Another important method for pest control is chemical control. Pesticides are useful because they quickly kill a significant portion of a pest population and they sometimes provide the only feasible method for preventing economic loss. Such different pest control tactics should work together rather than against each other to accomplish successful pest population control [11].

S. Zhang and D. Tan [13] investigated complex dynamics of Holling type II three species food chain system with impulsive perturbations on the predator. Especially, they took an impulsive perturbation as a biological control. Now, we consider the following Holling type II food chain system with biological control on the predator and chemical controls on all species:

Received July 7, 2008.

2000 Mathematics Subject Classification. Primary 34A37, 34D23, 34H05, 92D25.

Key words and phrases. Holling type II food chain system, biological and chemical controls, impulsive differential equation, Floquet theory. 
(1.1)

$$
\left\{\begin{array}{l}
x^{\prime}(t)=x(t)(a-b x(t))-\frac{c_{1} x(t) y(t)}{e_{1}+x(t)} \\
y^{\prime}(t)=-d_{1} y(t)+\frac{c_{2} x(t) y(t)}{e_{1}+x(t)}-\frac{c_{3} y(t) z(t)}{e_{2}+y(t)}, \\
z^{\prime}(t)=-d_{2} z(t)+\frac{c_{4} y(t) z(t)}{e_{2}+y(t)} \\
x\left(t^{+}\right)=\left(1-p_{1}\right) x(t), \\
y\left(t^{+}\right)=\left(1-p_{2}\right) y(t), \\
z\left(t^{+}\right)=\left(1-p_{3}\right) z(t), \\
x\left(t^{+}\right)=x(t) \\
y\left(t^{+}\right)=y(t)+q, \\
z\left(t^{+}\right)=z(t), \\
\left(x\left(0^{+}\right), y\left(0^{+}\right), z\left(0^{+}\right)\right)=\left(x_{0}, y_{0}, z_{0}\right),
\end{array}\right.
$$

where $T$ is the period of the impulsive immigration or stock of the predator, $0 \leq p_{1}, p_{2}, p_{3}<1$ present the fraction of the prey, predator and top predator which die due to the harvesting or pesticides etc and $q$ is the size of immigration or stock of the predator. Recently, it is of great interest to study dynamical properties for impulsive perturbations in population dynamics $[8,7,6,12,13$, $14]$.

In the next section, we introduce some notations used in this paper. In Section 3, we show the boundedness of the system and show the local stability of prey(pest)-free periodic solutions. Furthermore, we establish sufficient conditions for the permanence of the system (1.1) by using the Floquet theory and small perturbation skills.

\section{Preliminaries}

First, we shall introduce a few notations and definitions together with a few auxiliary results relating to comparison theorem, which will be useful for our main results.

Let $\mathbb{R}_{+}=[0, \infty)$ and $\mathbb{R}_{+}^{3}=\left\{\mathbf{x}=(x(t), y(t), z(t)) \in \mathbb{R}^{3}: x(t), y(t), z(t) \geq\right.$ $0\}$. Denote $\mathbb{N}$ the set of all of nonnegative integers, $\mathbb{R}_{+}^{*}=(0, \infty)$ and $f=$ $\left(f_{1}, f_{2}, f_{3}\right)^{T}$ the right hand of the first three equations in (1.1). Let $V: \mathbb{R}_{+} \times$ $\mathbb{R}_{+}^{3} \rightarrow \mathbb{R}_{+}$. Then $V$ is said to be in a class $V_{0}$ if

(1) $V$ is continuous on $(n T,(n+1) T] \times \mathbb{R}_{+}^{3}$, and $\lim _{(t, \mathbf{y}) \rightarrow(n T, \mathbf{x}) t>n T} V(t, \mathbf{y})$ $=V\left(n T^{+}, \mathbf{x}\right)$ exists.

(2) $V$ is a local Lipschitzian in $\mathbf{x}$.

Definition 2.1. For $V \in V_{0}$, we define the upper right Dini derivative of $V$ with respect to the impulsive differential system $(1.1)$ at $(t, \mathbf{x}) \in(n T,(n+1) T] \times \mathbb{R}_{+}^{3}$ 
by

$$
D^{+} V(t, \mathbf{x})=\limsup _{h \rightarrow 0+} \frac{1}{h}[V(t+h, \mathbf{x}+h f(t, \mathbf{x}))-V(t, \mathbf{x})] .
$$

Remark 2.2. (1) The solution of the system (1.1) is a piecewise continuous function $\mathbf{x}: \mathbb{R}_{+} \rightarrow \mathbb{R}_{+}^{3}, \mathbf{x}(t)$ is continuous on $(n T,(n+1) T], n \in \mathbb{N}$ and $\mathbf{x}\left(n T^{+}\right)=\lim _{t \rightarrow n T^{+}} \mathbf{x}(t)$ exists. (2) The smoothness properties of $f$ guarantee the global existence and uniqueness of solutions of the system (1.1). (See [5] for the details).

We will use a comparison result of impulsive differential inequalities. We suppose that $g: \mathbb{R}_{+} \times \mathbb{R}_{+} \rightarrow \mathbb{R}$ satisfies the following hypotheses:

(H) $g$ is continuous on $(n T,(n+1) T] \times \mathbb{R}_{+}$and the limit

$$
\lim _{(t, y) \rightarrow\left(n T^{+}, x\right)} g(t, y)=g\left(n T^{+}, x\right)
$$

exists and is finite for $x \in \mathbb{R}_{+}$and $n \in \mathbb{N}$.

Lemma $2.3([5])$. Suppose $V \in V_{0}$ and

$$
\left\{\begin{array}{l}
D^{+} V(t, \mathbf{x}) \leq g(t, V(t, \mathbf{x})), t \neq(n+\tau-1) T, n T \\
V\left(t, \mathbf{x}\left(t^{+}\right)\right) \leq \psi_{n}^{1}(V(t, \mathbf{x})), t=(n+\tau-1) T \\
V\left(t, \mathbf{x}\left(t^{+}\right)\right) \leq \psi_{n}^{2}(V(t, \mathbf{x})), t=n T
\end{array}\right.
$$

where $g: \mathbb{R}_{+} \times \mathbb{R}_{+} \rightarrow \mathbb{R}$ satisfies $(H)$ and $\psi_{n}^{1}, \psi_{+}^{2}: \mathbb{R}_{+} \rightarrow \mathbb{R}_{+}$are nondecreasing for all $n \in \mathbb{N}$. Let $r(t)$ be the maximal solution for the impulsive Cauchy problem

$$
\left\{\begin{array}{l}
u^{\prime}(t)=g(t, u(t)), t \neq(n+\tau-1) T, n T, \\
u\left(t^{+}\right)=\psi_{n}^{1}(u(t)), t=(n+\tau-1) T, \\
u\left(t^{+}\right)=\psi_{n}^{2}(u(t)), t=n T \\
u\left(0^{+}\right)=u_{0},
\end{array}\right.
$$

defined on $[0, \infty)$. Then $V\left(0^{+}, \mathbf{x}_{0}\right) \leq u_{0}$ implies that $V(t, \mathbf{x}(t)) \leq r(t), t \geq 0$, where $\mathbf{x}(t)$ is any solution of $(2.1)$.

We now indicate a special case of Lemma 2.3 which provides estimations for the solution of a system of differential inequalities. For this, we let $P C\left(\mathbb{R}_{+}, \mathbb{R}\right)$ $\left(P C^{1}\left(\mathbb{R}_{+}, \mathbb{R}\right)\right)$ denote the class of real piecewise continuous (real piecewise continuously differentiable) functions defined on $\mathbb{R}_{+}$.

Lemma $2.4([5])$. Let the function $u(t) \in P C^{1}\left(\mathbb{R}^{+}, \mathbb{R}\right)$ satisfy the inequalities

$$
\left\{\begin{aligned}
\frac{d u}{d t} & \leq f(t) u(t)+h(t), t \neq \tau_{k}, t>0 \\
u\left(\tau_{k}^{+}\right) & \leq \alpha_{k} u\left(\tau_{k}\right)+\beta_{k}, k \geq 0 \\
u\left(0^{+}\right) & \leq u_{0}
\end{aligned}\right.
$$


where $f, h \in P C\left(\mathbb{R}_{+}, \mathbb{R}\right)$ and $\alpha_{k} \geq 0, \beta_{k}$ and $u_{0}$ are constants and $\left(\tau_{k}\right)_{k \geq 0}$ is a strictly increasing sequence of positive real numbers. Then, for $t>0$,

$$
\begin{aligned}
u(t) \leq & u_{0}\left(\prod_{0<\tau_{k}<t} \alpha_{k}\right) \exp \left(\int_{0}^{t} f(s) d s\right) \\
& +\int_{0}^{t}\left(\prod_{0 \leq \tau_{k}<t} d_{k}\right) \exp \left(\int_{s}^{t} f(\gamma) d \gamma\right) h(s) d s \\
& +\sum_{0<\tau_{k}<t}\left(\prod_{\tau_{k}<\tau_{j}<t} d_{j}\right) \exp \left(\int_{\tau_{k}}^{t} f(\gamma) d \gamma\right) \beta_{k} .
\end{aligned}
$$

Similar result can be obtained when all conditions of the inequalities in the Lemmas 2.3 and 2.4 are reversed. Using Lemma 2.4, it is possible to prove that the solutions of the Cauchy problem (2.2) with strictly positive initial value remain strictly positive.

Lemma 2.5. The positive octant $\left(\mathbb{R}_{+}^{*}\right)^{3}$ is an invariant region for the system (1.1).

Proof. Let $(x(t), y(t), z(t)):\left[0, t_{0}\right) \rightarrow \mathbb{R}^{2}$ be a saturated solution of the system (1.1) with a strictly positive initial value $(x(0), y(0), z(0))$. By Lemma 2.4, we can obtain that, for $0 \leq t<t_{0}$,

$$
\left\{\begin{array}{l}
x(t) \leq x(0)\left(1-p_{1}\right)^{\left[\frac{t}{T}\right]} \exp \left(\int_{0}^{t} f_{1}(s) d s\right), \\
y(t) \leq y(0)\left(1-p_{2}\right)^{\left[\frac{t}{T}\right]} \exp \left(\int_{0}^{t} f_{2}(s) d s\right), \\
z(t) \leq z(0)\left(1-p_{3}\right)^{\left[\frac{t}{T}\right]} \exp \left(\int_{0}^{t} f_{3}(s) d s\right),
\end{array}\right.
$$

where $f_{1}(s)=a-b x(s), f_{2}(s)=-d_{1}+\frac{c_{2} x(s)}{e_{1}+x(s)}$ and $f_{3}(s)=-d_{2}+\frac{c_{4} y(s)}{e_{2}}$. Thus, $x(t), y(t), z(t)$ remain strictly positive on $\left[0, t_{0}\right)$.

Now, we give the basic properties of another impulsive differential equation as follows:

$$
\left\{\begin{array}{l}
y^{\prime}(t)=-d_{1} y(t), t \neq n T, t \neq(n+\tau-1) T \\
y\left(t^{+}\right)=\left(1-p_{2}\right) y(t), t=(n+\tau-1) T \\
y\left(t^{+}\right)=y(t)+q, t=n T
\end{array}\right.
$$

The system (2.5) is a periodically forced linear system. It is easy to obtain that (2.6) $y^{*}(t)=\left\{\begin{array}{l}\frac{q \exp \left(-d_{1}(t-(n-1) T)\right)}{1-\left(1-p_{2}\right) \exp \left(-d_{1} T\right)},(n-1) T<t \leq(n+\tau-1) T, \\ \frac{q\left(1-p_{2}\right) \exp \left(-d_{1}(t-(n-1) T)\right)}{1-\left(1-p_{2}\right) \exp \left(-d_{1} T\right)},(n+\tau-1) T<t \leq n T,\end{array}\right.$ 
$y^{*}\left(0^{+}\right)=y^{*}\left(n T^{+}\right)=\frac{q}{1-\left(1-p_{2}\right) \exp \left(-d_{1} T\right)}, y^{*}\left((n+\tau-1) T^{+}\right)=\frac{q\left(1-p_{2}\right) \exp \left(-d_{1} \tau T\right)}{1-\left(1-p_{2}\right) \exp \left(-d_{1} T\right)}$ is a positive periodic solution of (2.5). Moreover, we can obtain that

$$
y(t)=\left\{\begin{array}{l}
\left(1-p_{2}\right)^{n-1}\left(y\left(0^{+}\right)-\frac{q\left(1-p_{2}\right) e^{-T}}{1-\left(1-p_{2}\right) \exp \left(-d_{1} T\right)}\right) \exp \left(-d_{1} t\right)+y^{*}(t), \\
(n-1) T<t \leq(n+\tau-1) T, \\
\left(1-p_{2}\right)^{n}\left(y\left(0^{+}\right)-\frac{q\left(1-p_{2}\right) e^{-T}}{1-\left(1-p_{2}\right) \exp \left(-d_{1} T\right)}\right) \exp \left(-d_{1} t\right)+y^{*}(t), \\
(n+\tau-1) T<t \leq n T,
\end{array}\right.
$$

is a solution of (2.5). From (2.6) and (2.7), we get easily the following result.

Lemma 2.6. All solutions $y(t)$ of (2.5) tend to $y^{*}(t)$, i.e., $\left|y(t)-y^{*}(t)\right| \rightarrow 0$ as $t \rightarrow \infty$.

It follows from Lemma 2.6 that the general solution $y(t)$ of $(2.5)$ can be synchronized with the positive periodic solution $y^{*}(t)$ of (2.5) and we can obtain the complete expression for the prey and top predator free periodic solution of the system (1.1)

$$
\left(0, y^{*}(t), 0\right)
$$

\section{Main results}

First, we show that all solutions of (1.1) are uniformly bounded.

Theorem 3.1. There is an $M>0$ such that $x(t) \leq M, y(t) \leq M$ and $z(t) \leq M$ for all $t$ large enough, where $(x(t), y(t), z(t))$ is a solution of the system (1.1).

Proof. Let $(x(t), y(t), z(t))$ be a solution of $(1.1)$ and let $u(t)=\frac{c_{2}}{c_{1}} x(t)+y(t)+$ $\frac{c_{3}}{c_{4}} z(t)$ for $t \geq 0$. Then, if $t \neq n T, t \neq(n+\tau-1) T$ and $t>0$, then we obtain that $\frac{d u(t)}{d t}=-\frac{c_{2} b}{c_{1}} x^{2}(t)+\frac{c_{2} a}{c_{1}} x(t)-d_{1} y(t)-\frac{c_{3} d_{2}}{c_{4}} z(t)$ and hence $\frac{d u(t)}{d t}+$ $\beta_{0} u(t)=-\frac{c_{2} b}{c_{1}} x^{2}(t)+\frac{c_{2} a}{c_{1}} x(t)+\left(\beta-d_{1}\right) y(t)+\frac{c_{3}}{c_{4}}\left(\beta-d_{2}\right) z(t)$. From choosing $0<\beta_{0}<\min \left\{d_{1}, d_{2}\right\}$, we have

$$
\begin{aligned}
& \frac{d u(t)}{d t}+\beta_{0} u(t) \\
\leq & -\frac{c_{2} b}{c_{1}} x^{2}(t)+\frac{c_{2}}{c_{1}}\left(a+\beta_{0}\right) x(t), t \neq n T, t \neq(n+\tau-1) T, t>0 .
\end{aligned}
$$

As the right-hand side of (3.1) is bounded from above by $M_{0}=\frac{c_{2}\left(a+\beta_{0}\right)^{2}}{4 b^{2} c_{1}}$, it follows that

$$
\frac{d u(t)}{d t}+\beta_{0} u(t) \leq M_{0}, t \neq n T, n \neq(n+\tau-1) T, t>0 .
$$


If $t=n T$, then $u\left(t^{+}\right)=u(t)+q$ and if $t=(n+\tau-1) T$, then $u\left(t^{+}\right) \leq(1-p) u(t)$, where $p=\min \left\{p_{1}, p_{2}, p_{3}\right\}$. From Lemma 2.4, we get that

$$
\begin{aligned}
u(t) \leq & u_{0}\left(\prod_{0<k T<t}(1-p)\right) \exp \left(\int_{0}^{t}-\beta_{0} d s\right) \\
& +\int_{0}^{t}\left(\prod_{0 \leq k T<t}(1-p)\right) \exp \left(\int_{s}^{t}-\beta_{0} d \gamma\right) M_{0} d s \\
& +\sum_{0<k T<t}\left(\prod_{k T<j T<t}(1-p)\right) \exp \left(\int_{k T}^{t}-\beta_{0} d \gamma\right) q \\
\leq & u\left(0^{+}\right) \exp \left(-\beta_{0} t\right)+\frac{M_{0}}{\beta_{0}}\left(1-\exp \left(-\beta_{0} t\right)\right)+\frac{q \exp \left(\beta_{0} T\right)}{\exp \left(\beta_{0} T\right)-1}
\end{aligned}
$$

Since the limit of the right-hand side of (3.2) as $t \rightarrow \infty$ is

$$
\frac{M_{0}}{\beta_{0}}+\frac{q \exp \left(\beta_{0} T\right)}{\exp \left(\beta_{0} T\right)-1}<\infty
$$

it easily follows that $u(t)$ is bounded for sufficiently large $t$. Therefore, $x(t), y(t)$ and $z(t)$ are bounded by a constant $M$ for sufficiently large $t$.

Theorem 3.2. The periodic solution $\left(0, y^{*}(t), 0\right)$ is locally asymptotically stable if

$$
a T+\ln \left(1-p_{1}\right)<\frac{c_{1} q\left(\Gamma-e_{2} p_{2} \exp \left(-d_{1} \tau T\right)\right)}{e_{1} d_{1} \Gamma}
$$

and

$$
\frac{\left(\Gamma+q \exp \left(-d_{1} \tau T\right)\right)\left(\Gamma+q\left(1-p_{2}\right) \exp \left(-d_{1} T\right)\right)}{(\Gamma+q)\left(\Gamma+q\left(1-p_{2}\right) \exp \left(-d_{1} \tau T\right)\right)}>\left(1-p_{3}\right)^{\frac{d_{1}}{c_{4}}} \exp \left(-\frac{d_{1} d_{2} T}{c_{4}}\right),
$$

where $\Gamma=e_{2}\left(1-\left(1-p_{2}\right) \exp \left(-d_{1} T\right)\right)$.

Proof. The local stability of the periodic solution $\left(0, y^{*}(t), 0\right)$ of the system $(1.1)$ may be determined by considering the behavior of small amplitude perturbations of the solution. Let $(x(t), y(t), z(t))$ be any solution of the system (1.1). Define $u(t)=x(t), v(t)=y(t)-y^{*}(t), w(t)=z(t)$. Then they may be written as

where $\Phi(t)$ satisfies

$$
\left(\begin{array}{c}
u(t) \\
v(t) \\
w(t)
\end{array}\right)=\Phi(t)\left(\begin{array}{c}
u(0) \\
v(0) \\
w(0)
\end{array}\right),
$$

$$
\frac{d \Phi}{d t}=\left(\begin{array}{ccc}
a-\frac{c_{1}}{e_{1}} y^{*}(t) & 0 & 0 \\
\frac{c_{2}}{e_{1}} y^{*}(t) & -d_{1} & -\frac{c_{3} y^{*}(t)}{e_{2}+y^{*}(t)} \\
0 & 0 & -d_{2}+\frac{c_{4} y^{*}(t)}{e_{2}+y^{*}(t)}
\end{array}\right) \Phi(t)
$$


and $\Phi(0)=I$, the identity matrix. So the fundamental solution matrix is

$$
\Phi(t)=\left(\begin{array}{ccc}
\exp \left(\int_{0}^{t} a-\frac{c_{1}}{e_{1}} y^{*}(s) d s\right) & 0 & 0 \\
\exp \left(\int_{0}^{t} \frac{c_{2}}{e_{1}} y^{*}(s) d s\right) & \exp \left(-d_{1} t\right) & \exp \left(-\int_{0}^{t} \frac{c_{3} y^{*}(s)}{e_{2}+y^{*}(s)} d s\right) \\
0 & 0 & \exp \left(\int_{0}^{t}-d_{2}+\frac{c_{4} y^{*}(s)}{e_{2}+y^{*}(s)} d s\right)
\end{array}\right)
$$

The resetting impulsive conditions of the system (1.1) become

$$
\left(\begin{array}{l}
u\left((n+\tau-1) T^{+}\right) \\
v\left((n+\tau-1) T^{+}\right) \\
u\left((n+\tau-1) T^{+}\right)
\end{array}\right)=\left(\begin{array}{ccc}
1-p_{1} & 0 & 0 \\
0 & 1-p_{2} & 0 \\
0 & 0 & 1-p_{3}
\end{array}\right)\left(\begin{array}{c}
u((n+\tau-1) T) \\
v((n+\tau-1) T) \\
w((n+\tau-1) T)
\end{array}\right)
$$

and

Note that all eigenvalues of

$$
\left(\begin{array}{c}
u\left(n T^{+}\right) \\
v\left(n T^{+}\right) \\
w\left(n T^{+}\right)
\end{array}\right)=\left(\begin{array}{lll}
1 & 0 & 0 \\
0 & 1 & 0 \\
0 & 0 & 1
\end{array}\right)\left(\begin{array}{c}
u(n T) \\
v(n T) \\
w(n T)
\end{array}\right)
$$

$$
S=\left(\begin{array}{ccc}
1-p_{1} & 0 & 0 \\
0 & 1-p_{2} & 0 \\
0 & 0 & 1-p_{3}
\end{array}\right)\left(\begin{array}{ccc}
1 & 0 & 0 \\
0 & 1 & 0 \\
0 & 0 & 1
\end{array}\right) \Phi(T)
$$

are $\mu_{1}=\left(1-p_{1}\right) \exp \left(\int_{0}^{T} a-\frac{c_{1}}{e_{1}} y^{*}(t) d t\right), \mu_{2}=\left(1-p_{2}\right) \exp \left(-d_{1} T\right)<0$ and $\mu_{3}=$ $\left(1-p_{3}\right) \exp \left(\int_{0}^{T}-d_{2}+\frac{c_{4} y^{*}(t)}{e_{2}+y^{*}(t)} d t\right)$. Since $y^{*}(t)=\frac{q \exp \left(-d_{1} t\right)}{1-\left(1-p_{2}\right) \exp \left(-d_{1} T\right)}, 0<t \leq \tau T$, and $y^{*}(t)=\frac{q\left(1-p_{2}\right) \exp \left(-d_{1} t\right)}{1-\left(1-p_{2}\right) \exp \left(-d_{1} T\right)}, \tau T<t \leq T$, we have

$$
\int_{0}^{T} y^{*}(t) d t=\frac{q\left(\Gamma-e_{2} p_{2} \exp \left(-d_{1} \tau T\right)\right)}{d_{1} \Gamma}
$$

and

$$
\begin{aligned}
\int_{0}^{T} \frac{y^{*}(t)}{e_{2}+y^{*}(t)} d t & =\int_{0}^{\tau T} \frac{y^{*}(t)}{e_{2}+y^{*}(t)} d t+\int_{\tau T}^{T} \frac{y^{*}(t)}{e_{2}+y^{*}(t)} d t \\
& =-\frac{1}{d_{1}} \ln \left(\frac{\left(\Gamma+q \exp \left(-d_{1} \tau T\right)\right)\left(\Gamma+q\left(1-p_{2}\right) \exp \left(-d_{1} T\right)\right)}{(\Gamma+q)\left(\Gamma+q\left(1-p_{2}\right) \exp \left(-d_{1} \tau T\right)\right)}\right)
\end{aligned}
$$

where $\Gamma=e_{2}\left(1-\left(1-p_{2}\right) \exp \left(-d_{1} T\right)\right)$. It follows from (3.5) and (3.6) that the conditions $\left|\mu_{1}\right|<1$ and $\left|\mu_{3}\right|<1$ are equivalent to the equations (3.3) and (3.4), respectively. Therefore, from the Floquet theory [1], we obtain $\left(0, y^{*}(t), 0\right)$ is locally stable.

Definition 3.3. The system (1.1) is permanent if there exist $M \geq m>0$ such that, for any solution $(x(t), y(t), z(t))$ of the system (1.1) with $x_{0}, y_{0}, z_{0}>0$,

$$
\begin{aligned}
& m \leq \lim _{t \rightarrow \infty} \inf x(t) \leq \lim _{t \rightarrow \infty} \sup x(t) \leq M, \\
& m \leq \lim _{t \rightarrow \infty} \inf y(t) \leq \lim _{t \rightarrow \infty} \sup y(t) \leq M,
\end{aligned}
$$


and

$$
m \leq \lim _{t \rightarrow \infty} \inf z(t) \leq \lim _{t \rightarrow \infty} \sup z(t) \leq M
$$

To prove the permanence of the system (1.1), we consider the following two subsystems. If the top-predator is absent, i.e., $z(t)=0$, then the system (1.1) can be expressed as

$$
\left\{\begin{array}{l}
x^{\prime}(t)=x(t)(a-b x(t))-\frac{c_{1} x(t) y(t)}{e_{1}+x(t)}, \\
y^{\prime}(t)=-d_{1} y(t)+\frac{c_{2} x(t) y(t)}{e_{1}+x(t)}, \\
x\left(t^{+}\right)=\left(1-p_{1}\right) x(t), \\
y\left(t^{+}\right)=\left(1-p_{2}\right) y(t), \\
x\left(t^{+}\right)=x(t), \\
y\left(t^{+}\right)=y(t)+p, \\
\left(x\left(0^{+}\right), y\left(0^{+}\right)\right)=\left(x_{0}, y_{0}\right)
\end{array}\right.
$$

If the prey is extinct, then the system (1.1) can be expressed as

$$
\left\{\begin{array}{l}
y^{\prime}(t)=-d_{1} y(t)-\frac{c_{3} y(t) z(t)}{e_{2}+y(t)}, \\
z^{\prime}(t)=-d_{2} z(t)+\frac{c_{4} y(t) z(t)}{e_{2}+y(t)}, \\
y\left(t^{+}\right)=\left(1-p_{2}\right) y(t), \\
z\left(t^{+}\right)=\left(1-p_{3}\right) z(t), \\
y\left(t^{+}\right)=y(t)+p, \\
z\left(t^{+}\right)=z(t), \\
\left(y\left(0^{+}\right), z\left(0^{+}\right)\right)=\left(y_{0}, z_{0}\right) .
\end{array} t=n T+1\right) t
$$

Especially, B. Liu et al. [7] gave a condition for permanence of the subsystem (3.7).

Theorem 3.4 ([7]). The subsystem (3.7) is permanent if

$$
a T+\ln \left(1-p_{1}\right)>\frac{c_{1} q\left(\Gamma-e_{2} p_{2} \exp \left(-d_{1} \tau T\right)\right)}{e_{1} d_{1} \Gamma},
$$

where $\Gamma=e_{2}\left(1-\left(1-p_{2}\right) \exp \left(-d_{1} T\right)\right)$.

Theorem 3.5. The subsystem (3.8) is permanent if

$$
\frac{\left(\Gamma+q \exp \left(-d_{1} \tau T\right)\right)\left(\Gamma+q\left(1-p_{2}\right) \exp \left(-d_{1} T\right)\right)}{(\Gamma+q)\left(\Gamma+q\left(1-p_{2}\right) \exp \left(-d_{1} \tau T\right)\right)}<\left(1-p_{3}\right)^{\frac{d_{1}}{c_{4}}} \exp \left(-\frac{d_{1} d_{2} T}{c_{4}}\right),
$$

where $\Gamma=e_{2}\left(1-\left(1-p_{2}\right) \exp \left(-d_{1} T\right)\right)$. 
Proof. Let $(y(t), z(t))$ be a solution of the subsystem (3.8) with $y_{0}>0$ and $z_{0}>0$. From Theorem 3.1, we may assume that $y(t) \leq M$ and $z(t) \leq \frac{e_{2}}{c_{3}} M$. Then $y^{\prime}(t) \geq-\left(d_{1}+M\right) y(t)$. From Lemmas 2.3 and 2.6, we have $y(t) \geq u^{*}(t)-\epsilon$ for $\epsilon>0$, where

$$
u^{*}(t)=\left\{\begin{array}{l}
\frac{q \exp \left(-\left(d_{1}+M\right)(t-(n-1) T)\right)}{1-\left(1-p_{2}\right) \exp \left(-\left(d_{1}+M\right) T\right)},(n-1) T<t \leq(n+\tau-1) T, \\
\frac{q\left(1-p_{2}\right) \exp \left(-\left(d_{1}+M\right)(t-(n-1) T)\right)}{1-\left(1-p_{2}\right) \exp \left(-\left(d_{1}+M\right) T\right)},(n+\tau-1) T<t \leq n T .
\end{array}\right.
$$

Thus, we obtain that $y(t) \geq \frac{q\left(\exp \left(-\left(d_{1}+M\right) T\right)\right.}{1-\left(1-p_{2}\right) \exp \left(-\left(d_{1}+M\right) T\right)}-\epsilon \equiv m_{0}$ for sufficiently large $t$. Therefore, we only need to find an $m_{2}>0$ such that $z(t) \geq m_{2}$ for large enough $t$. We will do this in the following two steps.

(Step1) From the assumption of this theorem, we can choose $m_{1}>0, \epsilon_{1}>0$ small enough such that

$$
\Phi \equiv\left(1-p_{3}\right) \exp \left(-d_{2} T-\frac{c_{4}}{e_{2}} \epsilon_{1} T-\frac{c_{4}}{d_{1}} \ln \left(\frac{\Delta_{1}}{\Delta_{2}}\right)\right)>1,
$$

where $\Delta_{1}=\left(\gamma+q \exp \left(-\left(d_{1}+\frac{c_{3}}{e_{2}} m_{1}\right) \tau T\right)\right)\left(\gamma+q\left(1-p_{2}\right) \exp \left(-\left(d_{1}+\frac{c_{3}}{e_{2}} m_{1}\right) T\right)\right)$, $\left.\Delta_{2}=\gamma+q\right)\left(\gamma+q\left(1-p_{2}\right) \exp \left(-\left(d_{1}+\frac{c_{3}}{e_{2}} m_{1}\right) \tau T\right)\right.$ and $\gamma=\left(e_{2}+\epsilon_{1}\right)(1-(1-$ $\left.\left.p_{2}\right) \exp \left(-\left(d_{1}+\frac{c_{3}}{e_{2}} m_{1}\right) T\right)\right)$. In this step, we will show that $z\left(t_{1}\right) \geq m_{1}$ for some $t_{1}>0$. Suppose not, i.e., $z(t)<m_{1}$ for $t>0$. Consider the following system.

$$
\left\{\begin{aligned}
v^{\prime}(t) & =-\left(d_{1}+\frac{c_{3}}{e_{2}} m_{1}\right) v(t), \\
w^{\prime}(t) & =-\left(d_{2}-\frac{c_{4} v(t)}{e_{2}+v(t)}\right) w(t),
\end{aligned}\right\} t \neq(n+\tau-1) T, t \neq n T,
$$

Then, by Lemmas 2.3, we obtain $y(t) \geq v(t)$ and $z(t) \geq w(t)$. By Lemma 2.6, we have $v^{*}(t)+\epsilon_{1} \geq v(t) \geq v^{*}(t)-\epsilon_{1}$, where, for $t \in((n-1) T, n T]$,

$$
v^{*}(t)=\left\{\begin{array}{l}
\frac{q \exp \left(-\left(d_{1}+\frac{c_{3}}{e_{2}} m_{1}\right)(t-(n-1) T)\right)}{1-\left(1-p_{2}\right) \exp \left(-\left(d_{1}+\frac{c_{3}}{e_{2}} m_{1}\right) T\right)},(n-1) T<t \leq(n+\tau-1) T, \\
\frac{q\left(1-p_{2}\right) \exp \left(-\left(d_{1}+\frac{c_{3}}{e_{2}} m_{1}\right)(t-(n-1) T)\right)}{1-\left(1-p_{2}\right) \exp \left(-\left(d_{1}+\frac{c_{3}}{e_{2}} m_{1}\right) T\right)},(n+\tau-1) T<t \leq n T
\end{array}\right.
$$


is the periodic solution of the impulsive equation (2.5) with $d_{1}$ changed $d_{1}+$ $\frac{c_{3}}{e_{2}} m_{1}$. Thus

$$
\begin{aligned}
w^{\prime}(t) & \geq\left(-d_{2}+\frac{c_{4} v^{*}(t)-c_{4} \epsilon_{1}}{e_{2}+v^{*}(t)+\epsilon_{1}}\right) w(t) \\
& \geq\left(-d_{2}+\frac{c_{4} v^{*}(t)}{e_{2}+\epsilon_{1}+v^{*}(t)}-\frac{c_{4}}{e_{2}} \epsilon_{1}\right) w(t) .
\end{aligned}
$$

Integrating $(3.10)$ on $((n+\tau-1) T,(n+\tau) T]$, we get

$$
\begin{aligned}
& w((n+\tau) T) \\
\geq & w\left((n+\tau-1) T^{+}\right) \exp \left(\int_{(n+\tau-1) T}^{(n+\tau) T}-d_{2}-\frac{c_{4}}{e_{2}} \epsilon_{1}+\frac{c_{4} v^{*}(t)}{e_{2}+\epsilon_{1}+v^{*}(t)} d t\right) .
\end{aligned}
$$

Since

$$
\begin{aligned}
& \int_{(n+\tau-1) T}^{(n+\tau) T} \frac{v^{*}(t)}{e_{2}+\epsilon_{1}+v^{*}(t)} d t \\
= & -\frac{1}{d_{1}+\frac{c_{3}}{e_{2}} m_{1}} \int_{(\tau-1) T}^{T} \frac{\eta\left(1-p_{2}\right)}{e_{2}+\epsilon_{1}+\eta\left(1-p_{2}\right) x} d x-\frac{1}{d_{1}+\frac{c_{3}}{e_{2}} m_{1}} \int_{0}^{\tau T} \frac{\eta}{e_{2}+\epsilon_{1}+\eta x} d x,
\end{aligned}
$$

we get $w((n+\tau) T) \geq w((n+\tau-1) T) \Phi$. Therefore $z((n+\tau+k) T) \geq w((n+$ $\tau+k) T) \geq w((n+\tau) T) \Phi^{k} \rightarrow \infty$ as $k \rightarrow \infty$ which contradicts the boundedness of $z(t)$.

(Step 2) Without loss of generality, we may let $z\left(t_{1}\right)=m_{1}$. If $z(t) \geq m_{1}$ for all $t>t_{1}$, then the subsystem (3.8) is permanent. If not, we may let $t_{2}=\inf _{t>t_{1}}\left\{z(t)<m_{1}\right\}$. Then $z(t) \geq m_{1}$ for $t_{1} \leq t \leq t_{2}$ and, by continuity of $z(t)$, we have $z\left(t_{2}\right)=m_{1}$ and $t_{1}<t_{2}$. There exist a $t^{\prime}\left(>t_{2}\right)$ such that $z\left(t^{\prime}\right) \geq m_{1}$ by Step 1. Set $t_{3}=\inf _{t>t_{2}}\left\{z(t) \geq m_{1}\right\}$. Then $z(t)<m_{1}$ for $t_{2}<t<t_{3}$ and $z\left(t_{3}\right)=m_{1}$. We can continue this process by using Step 1 . If the process is stopped in finite times, we complete the proof. Otherwise, there exists an interval's sequence $\left[t_{2 k}, t_{2 k+1}\right], k \in \mathbb{N}$, which has the following property : $z(t)<m_{1}, t \in\left(t_{2 k}, t_{2 k+1}\right), t_{2 k-1}<t_{2 k} \leq t_{2 k+1}$ and $z\left(t_{n}\right)=m_{1}$, where $k, n \in \mathbb{N}$. Let $T_{0}=\sup \left\{t_{2 k+1}-t_{2 k} \mid k \in \mathbb{N}\right\}$. If $T_{0}=\infty$, then we can take a subsequence $\left\{t_{2 k_{i}}\right\}$ satisfying $t_{2 k_{i}+1}-t_{2 k_{i}} \rightarrow \infty$ as $k_{i} \rightarrow \infty$. As in the proof of the first step, this will lead to a contradiction to the boundedness of $z(t)$. Then we obtain $T_{0}<\infty$. Note that

$$
\begin{aligned}
z(t) & \geq z\left(t_{2 k}\right) \exp \left(\int_{t_{2 k}}^{t}-d_{2}-\frac{c_{4}}{e_{2}} \epsilon_{1}+\frac{c_{4} v^{*}(s)}{e_{2}+\epsilon_{1}+v^{*}(s)} d s\right) \\
& \geq m_{1} \exp \left(-d_{2} T_{0}\right) \equiv m_{2}, \quad t \in\left(t_{2 k}, t_{2 k+1}\right], k \in \mathbb{N} .
\end{aligned}
$$

Thus we obtain that $\liminf _{t \rightarrow \infty} z(t) \geq m_{2}$. Therefore we complete the proof. 
Theorem 3.6. The system (1.1) is permanent if

$$
a T+\ln \left(1-p_{1}\right)>\frac{c_{1} q\left(\Gamma-e_{2} p_{2} \exp \left(-d_{1} \tau T\right)\right)}{e_{1} d_{1} \Gamma}
$$

and

$$
\frac{\left(\Gamma+q \exp \left(-d_{1} \tau T\right)\right)\left(\Gamma+q\left(1-p_{2}\right) \exp \left(-d_{1} T\right)\right)}{(\Gamma+q)\left(\Gamma+q\left(1-p_{2}\right) \exp \left(-d_{1} \tau T\right)\right)}<\left(1-p_{3}\right)^{\frac{d_{1}}{c_{4}}} \exp \left(-\frac{d_{1} d_{2} T}{c_{4}}\right),
$$

where $\Gamma=e_{2}\left(1-\left(1-p_{2}\right) \exp \left(-d_{1} T\right)\right)$.

Proof. Let $\Gamma=e_{2}\left(1-\left(1-p_{2}\right) \exp \left(-d_{1} T\right)\right)$. Consider the following two subsystem of the system (1.1).

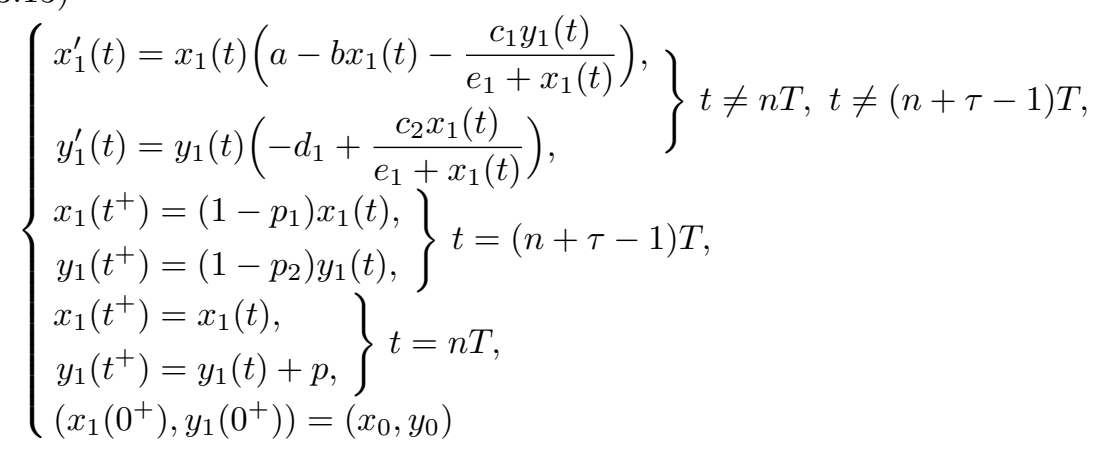

and

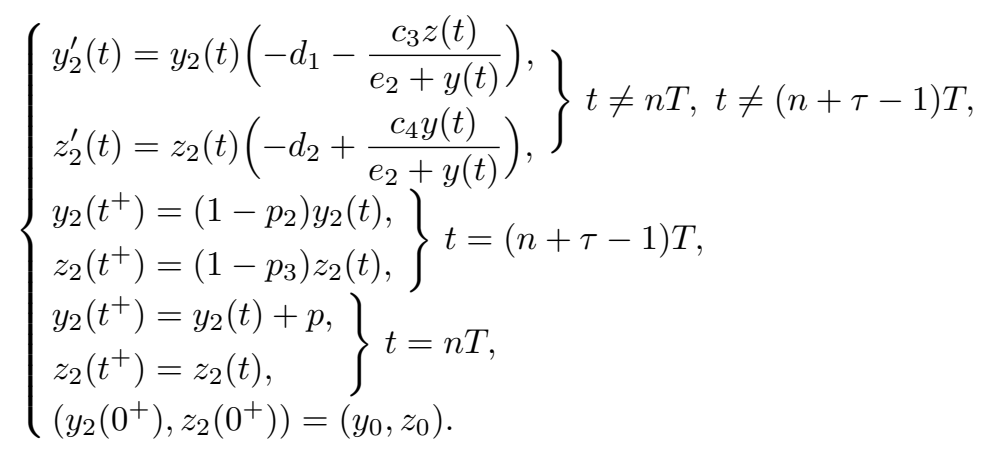

It follows from Lemma 2.3 that $x_{1}(t) \leq x(t), y_{1}(t) \geq y(t), y_{2}(t) \leq y(t)$ and $z_{2}(t) \leq z(t)$. If $a T+\ln \left(1-p_{1}\right)>\frac{c_{1} q\left(\bar{\Gamma}-e_{2} p_{2} \exp \left(-d_{1} \tau T\right)\right)}{e_{1} d_{1} \Gamma}$, by Theorem 3.4 the subsystem (3.13) is permanent. Thus we can take $T_{1}>0$ and $m_{1}>0$ such that $x(t) \geq m_{1}$ for $t \geq T_{1}$. Further, if $\frac{\left(\Gamma+q \exp \left(-d_{1} \tau T\right)\right)\left(\Gamma+q\left(1-p_{2}\right) \exp \left(-d_{1} T\right)\right)}{(\Gamma+q)\left(\Gamma+q\left(1-p_{2}\right) \exp \left(-d_{1} \tau T\right)\right)}<$ $\left(1-p_{3}\right)^{\frac{d_{1}}{c_{4}}} \exp \left(-\frac{d_{1} d_{2} T}{c_{4}}\right)$, by Theorem 3.5 the subsystem (3.14) is also permanent. Therefore, there exists $T_{2}>0$ and $m_{2}, m_{3}>0$ such that $y(t) \geq m_{2}$ and $z(t) \geq m_{3}$ for $t \geq T_{2}$. The proof is complete. 
Remark 3.7. It follow from Theorems 3.1, 3.2 and 3.6 that Theorems 3.1, 3.2 and 3.4 in [13] are Corollaries.

\section{References}

[1] D. D. Bainov and P. S. Simeonov, Impulsive Differential Equations: Periodic Solutions and Applications, vol. 66, of Pitman Monographs and Surveys in Pure and Applied Mathematics, Longman Science \& Technical, Harlo, UK, 1993.

[2] J. B. Collings, The effects of the functional response on the bifurcation behavior of a mite predator-prey interaction model, J. Math. Biol. 36 (1997), 149-168.

[3] S.-B. Hsu and T.-W. Huang, Global stability for a class of predator-prey systems, SIAM J. Appl. Math. 55 (1995), no. 3, 763-783.

[4] K. Kitamura, K. Kashiwagi, K.-I. Tainaka, T. Hayashi, J. Yoshimura, T. Kawai, and T. Kajiwara, Asymmetrical effect of migration on a prey-predator model, Physics Letters A 357 (2006), 213-217.

[5] V. Lakshmikantham, D. Bainov, and P.Simeonov, Theory of Impulsive Differential Equations, World Scientific Publisher, Singapore, 1989.

[6] X. Liu and L. Chen, Complex dynamics of Holling type II Lotka-Volterra predator-prey system with impulsive perturbations on the predator, Chaos, Solitons and Fractals 16 (2003), 311-320.

[7] B. Liu, Z. Teng, and L. Chen, Analsis of a predator-prey model with Holling II functional response concerning impulsive control strategy, J. of Comp. and Appl. Math. 193(2006), no. 1, 347-362.

[8] B. Liu, Y. Zhang, and L. Chen, Dynamic complexities in a Lotka-Volterra predator-prey model concerning impulsive control strategy, Int. J. of Bifur. and Chaos 15 (2005), no. 2, 517-531.

[9] E. Saez and E. Gonzalez-Olivares, Dynamics of a predator-prey model, SIAM J. Appl. Math. 59(1999), no. 5, 1867-1878.

[10] G. T. Skalski and J. F. Gilliam, Funtional responses with predator interference: viable alternatives to the Holling type II mode, Ecology 82 (2001), 3083-3092.

[11] S. Tang, Y. Xiao, L. Chen, and R. A. Cheke, Integrated pest management models and their dynamical behaviour, Bulletin of Math. Biol. 67 (2005), 115-135.

[12] S. Zhang and L. Chen, Chaos in three species food chain system with impulsive perturbations, Chaos Solitons and Fractals 24 (2005), 73-83.

[13] A Holling II functional response food chain model with impulsive perturbations, Chaos Solitons and Fractals 24 (2005), 1269-1278.

[14] S. Zhang, L. Dong, and L. Chen, The study of predator-prey system with defensive ability of prey and impulsive perturbations on the predator, Chaos, Solitons and Fractals 23 (2005), 631-643.

[15] S. Zhang, D. Tan, and L. Chen, Dynamic complexities of a food chain model with impulsive perturbations and Beddington-DeAngelis functional response, Chaos Solitons and Fractals 27 (2006), 768-777.

Department of Mathematics

KYUNGPOOK NATIONAL UNIVERSITY

DAEGU 702-701, KOREA

E-mail address: hkbaek@knu.ac.kr 